\title{
An Improved Fruit Fly Optimization Algorithm and Its Application
}

\author{
Shi HuiShu, a , San $\mathrm{Ye}^{1, \mathrm{~b}}$ and Zhu $\mathrm{Yi}^{1, \mathrm{c}}$ \\ ${ }^{1}$ Harbin Institute of Technology, Control and Simulation Center, Harbin, Heilongjiang, China \\ ashihuishu_10@126.com, banye@hit.edu.cn, c zhuyi@hit.edu.cn
}

Keywords: fruit fly optimization algorithm, intelligent algorithm, SVM, analog circuit fault diagnosis

\begin{abstract}
Fruit fly optimization algorithm is a new swarm intelligent algorithm proposed in recent years and has been concerned for its few parameters and high computational efficiency. However, the application of the algorithm is limited for unstable optimization capability. To solve that question, an improved fruit fly optimization algorithm is proposed in this paper. Some factors affecting the performance of the algorithm are improved. The improved algorithm are proved by function optimization and applied to the analog circuit fault diagnosis.
\end{abstract}

\section{Introduction}

Swarm intelligent algorithm has distributed and self-organizing features naturally and is good at nonlinear problems solution. The common swarm intelligent algorithms such as GA [1], PSO [2], ant colony optimization [3] and fish swarm algorithm [4], are widely used to solve complex nonlinear problems like pattern recognition, image processing and machine learning.

Fruit fly optimization algorithm (FOA) is a new swarm intelligent algorithm proposed by Pan in recent years [5]. It is easy to understand and has simple computation [6,7]. However, the thought of FOA decides it has some certain shortcomings like unstable optimization capability and easy to fall into local optimization. The shortcomings limit the application of the algorithm.

To improve applicability of FOA, an improved fruit fly optimization algorithm (IFOA) is proposed in this paper. Two factors affecting the search capability were improved. The optimization capability of IFOA was verified by Branin function, and the algorithm was used to optimize the analog circuit fault diagnosis model.

The main structure of this article is as follows: The first section introduces the research motivation of this paper. The second section introduces the standard fruit fly optimization algorithm and IFOA is proposed and tested in the third section. The application of the algorithm was shown in the fourth section. The research conclusion could be found in the last section.

\section{Standard Fruit Fly Optimization Algorithm}

FOA search global optimization based on the food finding behavior of the fruit fly. The fruit fly can find food by smell in distance. After get close to the food location it can find the location of food and the company's flocking by vision and fly towards that direction. The process is shown in Fig. 1.

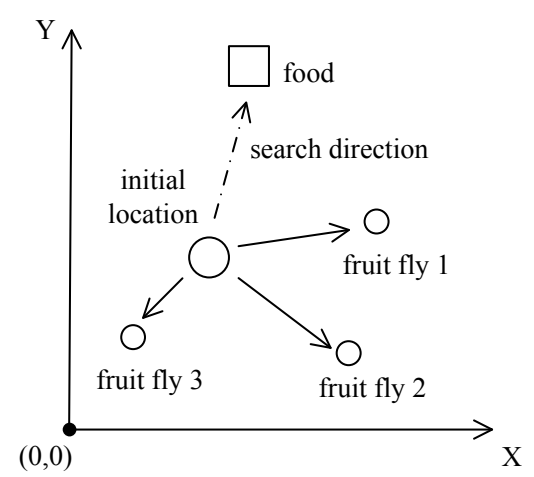

Figure 1 Food finding process of fruit fly swarm 
The steps of fruit fly algorithm are as follow.

1) Set the size of the fruit fly swarm and the initial location $\left(X_{0}, Y_{0}\right)$.

2) Give the direction and distance for the search of food to individual fruit fly randomly.

$$
X_{i}=X_{0}+\text { Random, } Y_{i}=Y_{0}+\text { Random }
$$

3) Calculate the distance to the origin $D$ and the smell concentration judgment value $S$ of each fruit fly.

$$
D_{i}=\sqrt{X_{i}^{2}+Y_{i}^{2}}, S_{i}=1 / D_{i}
$$

4) Find the smell concentration $\operatorname{Smell}_{i}$ of the individual location of the fruit fly by substituting of $S$ into smell concentration judgment value function (or called fitness function).

$$
\text { Smell }_{i}=\operatorname{fitness}\left(S_{i}\right)
$$

5) Take the fruit fly location with maximal smell concentration among the fruit fly swarm as the optimal location. The fruit fly swarm will fly towards that location by vision.

6) Repeat the implementation of steps 2-5. If the smell concentration is superior to the previous iterative smell concentration, retain the new optimal location. Otherwise, retain the optimal location of the previous generation.

\section{Improved Fruit Fly Optimization Algorithm}

Initial location, population size and flight distance are main factors which affect the convergence speed of fruit fly algorithm.

Usually, the larger the population size, the algorithm converges faster and the initial location close to the optimization will beneficial to the search efficiency of the algorithm.

The flight distance of fruit fly has a significant effect on optimization capability. A large distance is conducive to jump out of local optimization, but reduce local search capability; a small distance may fall into local optimization, and reduce search efficiency. That makes a fixed flight distance difficult to adapt the entire optimization process. In addition, the different parameters usually have different sensitivity to the optimal value. The same flight distance is obviously not suitable for all the parameters.

Aiming to the above problems, an improved fruit fly algorithm with a given initial location and a variable flight distance is proposed in this paper. First, the random initial location of fruit fly swarm is replaced by a given initial location. For improves the search capability and efficiency of the algorithm, the parameter scope is searched by uniform design, and then the optimal value is selected from experimental results to be the initial location. Second, the fixed flight distance is replaced by a variable flight distance to reduce the effect of parameter sensitivity. Make the flight distance correlated with the smell concentration, take large flight distance at first to improve search speed and small flight distance to enhance local search capability.

Initial Location Selection Based on Uniform Design. Uniform design is a direct experimental design method in statistics, which follows the principle of uniform dispersion [8]. The key of the method is to make most of uniform points tested, and the aim is to get the good result using less test points. As an effective way to reduce the amount of experiments, it successfully used in industry, agriculture, medicine, food and other fields.

The test scheme designed by uniform design has a set of representative and well-distributed test points, and the relationship between factors and results can be reflected by a small amount of experiment. The number of experiments is reduced significantly. Uniform design is suitable for test analysis of complex systems with multi-level and multi-factor. 
For IFOA, uniform design is used to search value scope to get the initial location of the fruit fly swarm. By giving an initial location with good value, search efficiency of the algorithm can be improved and the risk of falling into local optimization can be reduced.

Variable Flight Distance. As can be seen in Eq. 1, the flight distance is random. Let the interval of iterative step size is $[-r, r]$, express the random value as

$$
\text { Random }=2 r * \operatorname{rand}()-r
$$

which $\operatorname{rand}()$ is a pseudo-random number. Obviously, the flight distance of the fruit fly individual is controlled by the parameter $r$.

It is mentioned above that the flight distance of the fruit fly should be correlated with the parameters. From Eq. 2 and Eq. 3, there is

$$
-\frac{1}{S_{i}} \leq X_{i} \leq \frac{1}{S_{i}},-\frac{1}{S_{i}} \leq Y_{i} \leq \frac{1}{S_{i}}
$$

Simply, let

$$
r=k \cdot \frac{1}{S_{i}}
$$

and the random value scope is $\left[-\frac{k}{S_{i}}, \frac{k}{S_{i}}\right]$.

For the more sensitive parameters, let $k$ small. On the contrary, let $k$ large.

In the search process, adjust the parameter $k$ according to the smell concentration $S_{m e l l}$ and iteration times. Let

$$
k=\alpha \cdot k
$$

If the smell concentration $S_{m e l l}$ unchanged after $n$ times, let $\alpha>1$ to jump out of local optimal value. In the late of search process, let $0.5<\alpha<1$ to improve the local optimization capability.

The flowchart of IFOA is shown in Fig. 2.

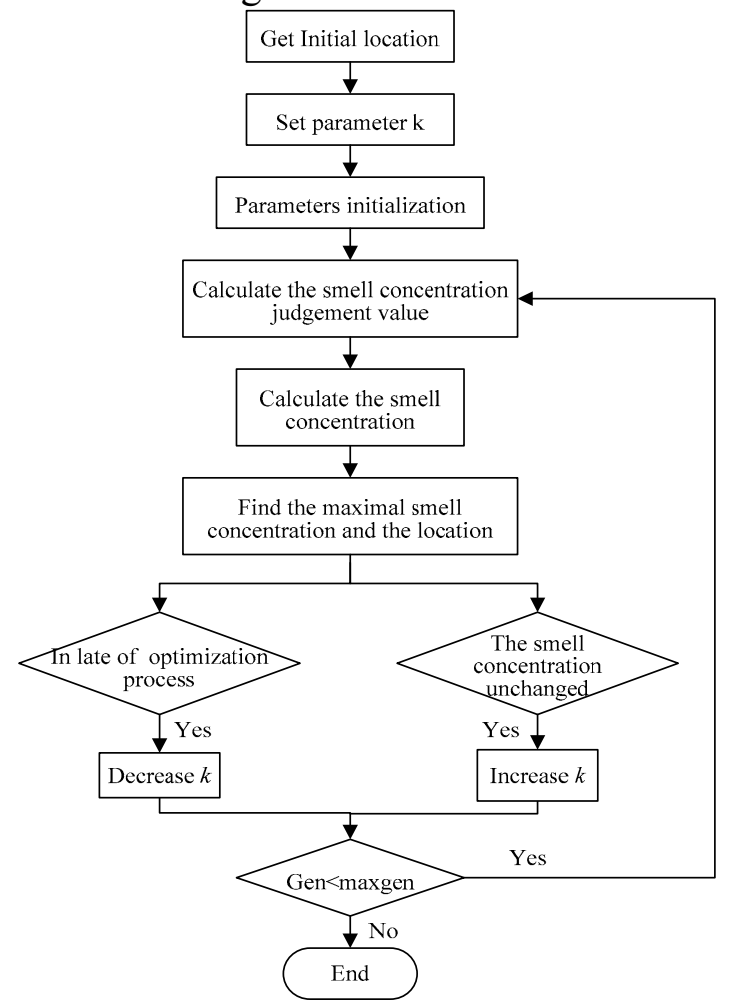

Figure 2 The flowchart of IFOA 
Validation of IFOA. Take Branin function as an example to verify the global optimization capability of IFOA, and compare with FOA.

Branin function is as follow.

$$
f=\left(x_{2}-\frac{5.1}{4 \pi^{2}} x_{1}^{2}+\frac{5}{\pi}-6\right)^{2}+10\left(1-\frac{1}{8 \pi}\right) \cos x_{1}+10
$$

which $-5 \leq x_{1} \leq 10,0 \leq x_{2} \leq 15$. The minimal value is $f_{\min }=0.3979$.

The minimal value was searched by FOA and IFOA respectively. Let the size of fruit fly swarm was 20 and iteration times was 1000. The initial location of FOA was random and the flight distance was $[-1,1]$. The initial location of IFOA was determination by the experimental result of uniform design. Divided the search scope of $x_{1}$ and $x_{2}$ into 6 levels, and computed according to the test scheme $U_{6}^{*}\left(6^{4}\right)$. The experiment shown that the function has minimal value $f=17.5524$ when $x_{1}=1$ and $x_{2}=3$. That point was considered as the initial location of the fruit fly swarm.

By analyzing Branin function, it can be found that $x_{1}$ affect the function value greatly, therefore, let $k_{1}=0.4, k_{2}=0.6$.

Run the experiment 30 times and put the average of optimal value (AOV) and the average of valid iterations (AVI) in Table 1.

Table $1 \mathrm{AVO}$ and AVI of FOA and IFOA

\begin{tabular}{|c|c|c|}
\hline & AVO & AVI \\
\hline FOA & 0.7366 & 467 \\
\hline IFOA & 0.3980 & 142 \\
\hline
\end{tabular}

As is shown in Table 1, AOV of IFOA is closer to the optimal value of Branin function and has less iteration. IFOA is superior to FOA on optimization capability, convergence rate and stability. AOV of FOA is significantly higher than AOV of IFOA, which is because FOA trapped into local optimization once. Remove the local optimization, AOV of FOA rise to 0.3995. The experiment shows that the algorithm proposed in this paper is effective.

The optimization process of FOA and IFOA are illustrated respectively in Fig. 3 and Fig. 4.
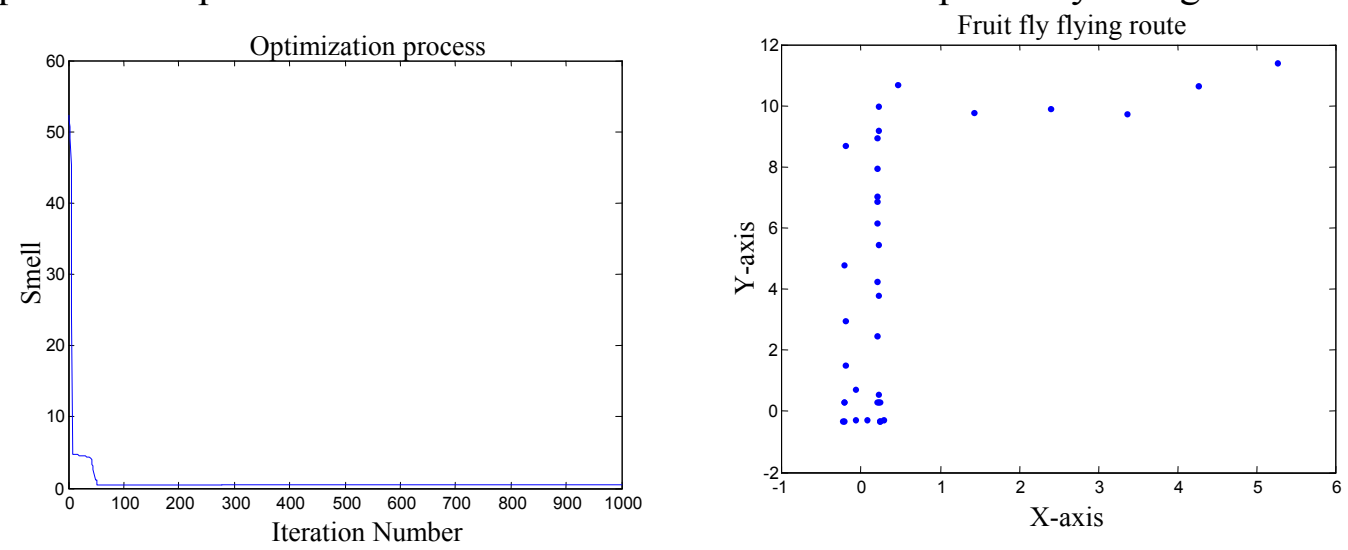

Figure 3 Optimization process of Branin function based on FOA

The smell concentration Smell ${ }_{i}$ (or known as function value) has significant change in Fig. 3 , it is caused by the change of the parameter $k$.

It should be noted that the two improvements proposed in this paper are mainly for practical application. Although the search optimization of function can reflect the optimization capability of the algorithm, the application value of the algorithm is hard to reflect completely. Therefore, the SVM model optimization of analog circuit fault diagnosis is considered as an example to illustrate the application value of IFOA in next section. 

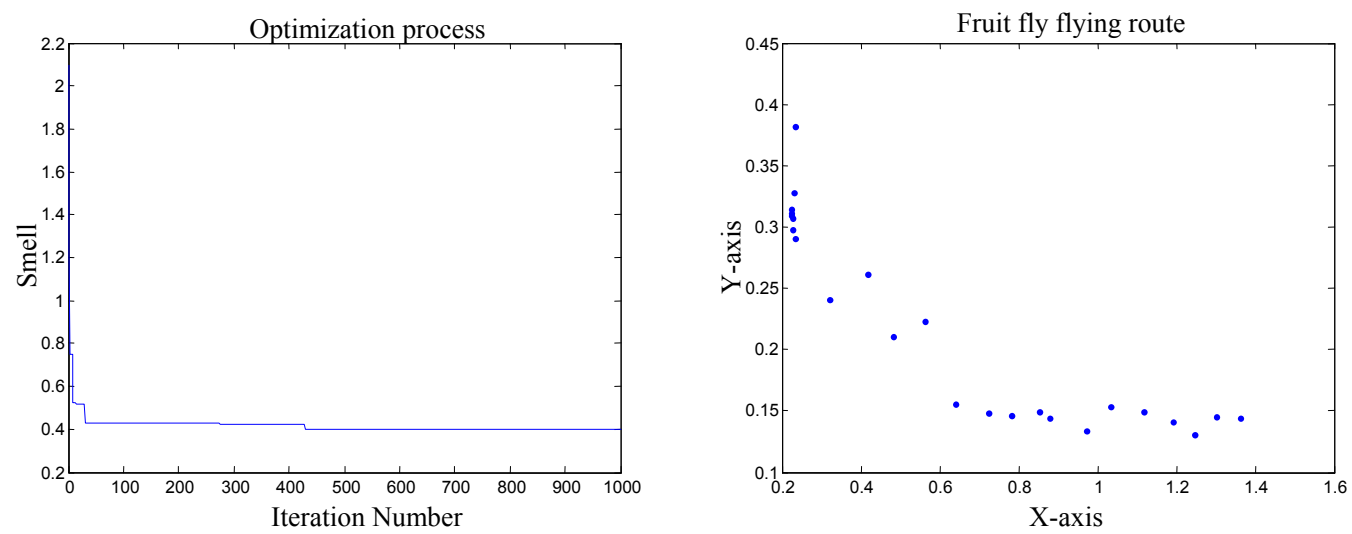

Figure 4 Optimization process of Branin function based on IFOA

\section{Application of IFOA on Analog Circuit Fault Diagnosis}

The importance of analog circuit fault diagnosis is increasing with the wide application of electronic equipment in aerospace, communication, energy, and daily life. SVM based on kernel method obtained widespread attention in analog circuit fault diagnosis, for it has excellent pattern classification capability and is suitable to solve the decision problem of limited samples.

The application value of method proposed in this article is illustrated by the soft fault diagnosis of Sallen-key band-pass filter circuit. The structure of the circuit is shown in Fig. 5.

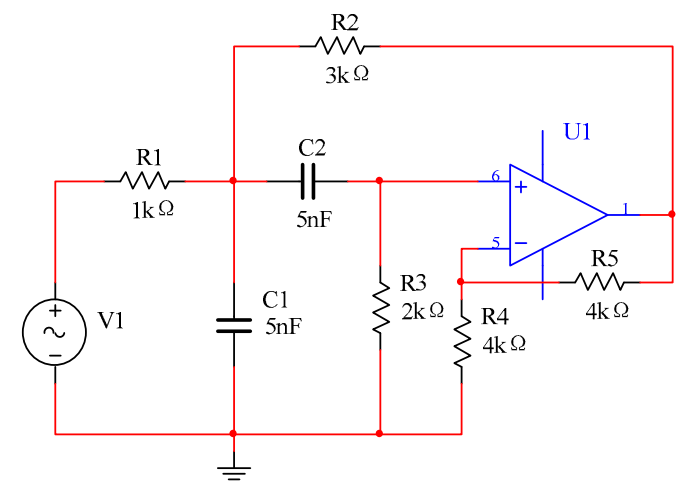

Figure 5 Sallen-key band-pass filter circuit

It can be found that the change of capacitor $C_{1}, C_{2}$ and resistor $R_{1}, R_{3}$ have significant effect on the circuit output by sensitivity analysis. There are 8 kind of soft faults on condition that each of the above elements parameter is positive skew 50\% and negative skew 50\% relative to nominal value. Soft fault data sets were constructed on that basis.

Monte-Carlo simulation was done 200 times respectively on the circuit in each above case, and fault sample set constructed by efficient point sampling. 120 times simulation results were taken as training set, and the others were taken as test set.

Let the search scope of $\gamma$ is $\left[2^{-8}, 2^{-6}, 2^{-4}, \cdots, 2^{6}\right]$ and the search scope of $C$ is $\left[2^{-4}, 2^{-2}, 2^{0}, \cdots, 2^{10}\right]$. Constituted SVM based on Gaussian kernel and optimized the model parameters by IFOA.

First, divided the search scope of $\gamma$ and $C$ into 8 levels and computed according to the test scheme $U_{8}^{*}\left(8^{5}\right)$ to get the initial location of the fruit fly swarm.

Second, set the other parameters. The population size was 20 and the iteration number was 100 . It can easily find that $\gamma$ is more sensitive, let $k=0.4$. For insensitive parameter $C$, let $k=0.8$.

The optimization process is shown in Fig. 6 . The classification accuracy on training set is $88.98 \%$, the classification accuracy on test set is $88.47 \%$ and training time is $142.92 \mathrm{~s}$.

It can be seen from the experimental results that IFOA can be applied to optimize the analog circuit fault diagnosis model effectively. The algorithm can be used to solve practical problems. 

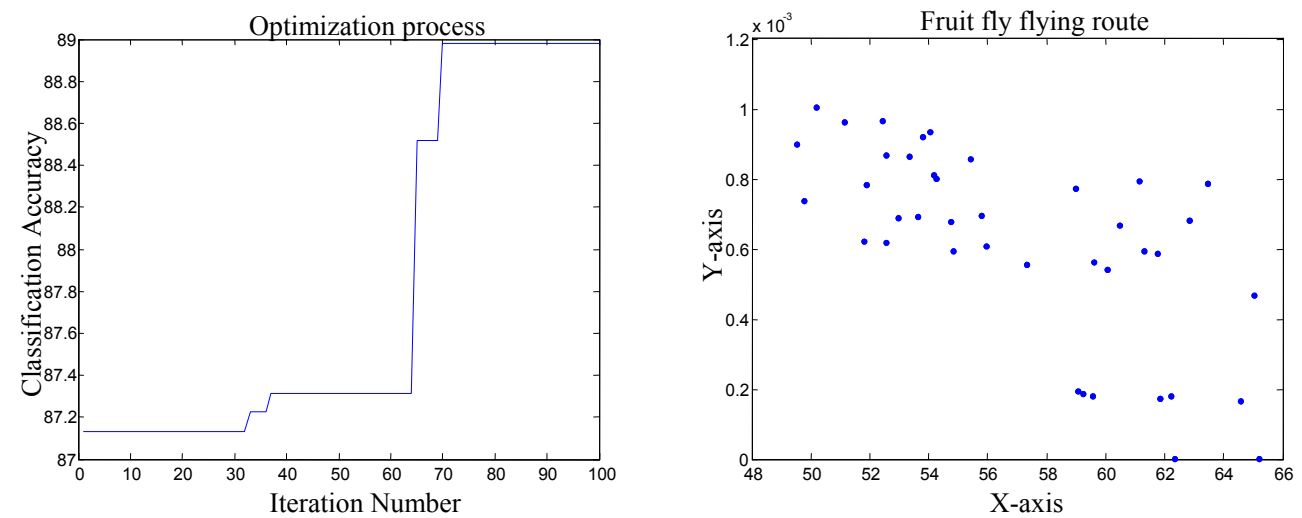

Figure 6 Optimization process of Gauss-SVM based on IFOA

\section{Conclusion}

As a new intelligent algorithm, fruit fly optimization algorithm has the disadvantages of easy to fall into local optimization and poor practicability and still need to improve.

Aiming to the problem, an improved fruit fly optimization algorithm is proposed in this paper. The selection of the initial location and flight distance of the fruit fly swarm is improved for stronger optimization capability and faster convergence rate.

The effectiveness of IFOA is proved by searching the optimal value of Branin function. By comparing with FOA, IFOA shows better stability and faster search speed. Then, the method was used to solve analog circuit fault diagnosis model optimization problem, and the application value of IFOA is proved. The experimental results show that IFOA is an effective intelligent optimization algorithm; it has good optimization capability and extensive application prospect.

\section{References}

[1] M.B. Aryanezhad and M. Hemati: A New Genetic Algorithm for Solving Nonconvex Nonlinear Programming Problems. Applied Mathematics and Computation. Vol. 199 (2008), p. 186-194

[2] Y.H. Shi and R.C. Eberhart: Empirical Study of Particle Swarm Optimization. IEEE International Conference on Evolutionary Computation. (1999), p. 1945-1950

[3] M. Dorigo and L.M. Gambardella: Ant Colony System: a Cooperative Learning Approach to The Traveling Salesman Problem. IEEE Transactions on Evolutionary Computation. Vol. 1 (1997), p. $53-66$

[4] X.L. Li, Z.J. Shao and J.X. Qian: An Optimizing Method Based on Autonomous Animats: Fish-swarm Algorithm. Systems Engineering-Theory and Practice. Vol. 22 (2002), p. 32-38

[5] W.T. Pan: A New Fruit Fly Optimization Algorithm: Taking The Financial Distress Model as An Example. Knowledge-Based Systems. Vol. 26 (2012), p. 69-74

[6] W.T. Pan: Using Fruit Fly Optimization Algorithm Optimized General Regression Neural Network to Construct The Operating Performance of Enterprises Model. Journal of Taiyuan University of Technology (Social Sciences Edition). Vol. 29 (2011), p. 1-5

[7] D. Shan, G.H. Cao and H.J. Dong: LGMS-FOA: An Improved Fruit Fly Optimization Algorithm for Solving Optimization Problems. Mathematical Problems in Engineering. Vol. 2013 (2013).

[8] K.T. Fang, M.Q. Liu and Y.D. Zhou: Experimental Design and Modeling. Chemical Industry Press. Beijing (2011). 